\title{
On the Effects of Different Interpretations of Stochastic Differential Equations
}

\author{
Claudio Floris \\ Department of Civil and Environmental Engineering, Politecnico di Milano, Milano, Italy \\ Email: claudio.floris@polimi.it
}

How to cite this paper: Floris, C. (2019) On the Effects of Different Interpretations of Stochastic Differential Equations. Applied Mathematics, 10, 876-891. https://doi.org/10.4236/am.2019.1011063

Received: September 13, 2019

Accepted: October 25, 2019

Published: October 28, 2019

Copyright (C 2019 by author(s) and Scientific Research Publishing Inc. This work is licensed under the Creative Commons Attribution International License (CC BY 4.0).

http://creativecommons.org/licenses/by/4.0/

\begin{abstract}
This paper addresses the problem of the interpretation of the stochastic differential equations (SDE). Even if from a theoretical point of view, there are infinite ways of interpreting them, in practice only Stratonovich's and Itô's interpretations and the kinetic form are important. Restricting the attention to the first two, they give rise to two different Fokker-Planck-Kolmogorov equations for the transition probability density function (PDF) of the solution. According to Stratonovich's interpretation, there is one more term in the drift, which is not present in the physical equation, the so-called spurious drift. This term is not present in Itô's interpretation so that the transition PDF's of the two interpretations are different. Several examples are shown in which the two solutions are strongly different. Thus, caution is needed when a physical phenomenon is modelled by a SDE. However, the meaning of the spurious drift remains unclear.
\end{abstract}

\section{Keywords}

Stochastic Differential Equations, White Noise Processes, Itô's Interpretation, Stratonovich's Interpretation

\section{Introduction}

From the beginning of the last century evidence became clear that a deterministic vision of the physical phenomena it is insufficient to describe them, and to foresee future occurrences as large uncertainties are always involved in real world phenomena. This line of thought has to be ascribed to Einstein [1], who gave an explanation to the Brownian motion. A few years after Einstein Langevin applied Newton's law to a Brownian particle [2], obtaining the same results as Einstein in a more straightforward way. In Langevin's study the resultant force caused by the collisions and acting on the particle was idealized as an ex- 
tremely irregular random force, the so-called white noise, a Gaussian process because of the central limit theorem. In this way, the displacement $X(t)$ of the particle is a random process. Fokker, Planck, and Kolmogorov independently found the partial differential equation that governs the time evolution of the probability density function (PDF) of $X(t)$ [3] [4] [5]. From then, this equation was called Fokker-Planck-Kolmogorov equation (FPK). Among the numerous other contributions on this subject we quote Wiener and Chandrasekhar [6] [7]. We shall call this line of thought as Langevin's description.

The other line of thought is quantum mechanics. In both lines the outcome of an experiment or of a phenomenon can no longer be foreseen precisely, but only the probability that it belongs to a set of events is calculable.

From the fifties, Langevin' description was applied in broad and broad fields of Physics, Engineering, Natural sciences and Medicine. When uncertainties are considered in studying a physical problem, the agencies entering the phenomenological equation are considered stochastic processes so that the equation becomes a stochastic differential equation (SDE). However, in the scientific and technical literature the name SDE is used to denote an equation which is acted by white noise stochastic processes, that is processes having a power spectral density constant on the frequency axis. Clearly, this is a mathematical idealization as it causes the independence of the values of the process in two instants $t_{j}$ and $t_{k}$ how much small the interval $t_{k}-t_{j}$ may be. incorporating the applicable criteria that follow.

For simplicity's sake, reference is made to a scalar SDE in which the excitation is a Gaussian white noise process. Writing down its solution, integrals involving the Brownian motion appear: this process is the parent of the Gaussian white noise as the latter is the derivative in the sense of the mathematical distributions of the former. If the Gaussian white noise acts externally (or additively), these integrals have a unique value. Unfortunately, in the case of a parametric (multiplicative) Gaussian white noise excitation the integral has infinite values depending on the position of the point in the discretization interval. Itô chose the inferior point of the interval [8], while Stratonovich chose the midpoint [9]. In the so-called kinetic interpretation the superior point is chosen [10] [11]

The coefficients of the FPK equation are named first and second derivate moment. The second derivate moment is the same in both Itô's and Stratonovich's interpretations, but the first is not so that two different FPK equations arise (for the computation of the derivate moments see Lin and Cai, [11], pages 127, 128, Cai and Zhu, [12], pages 117, 120). In general, the first derivate moment depends on the interpretation of the stochastic integral with respect to the Brownian motion.

The debate on which interpretation is preferable has been live, and it be considered to stand still today: [10] [11] [13]-[32]. Analyzing the literature, the following conclusions can be drawn: 1) It is confirmed that the solution of an SDE depends on how the stochastic integral is interpreted. 2) Itô's interpretation gives rise to simpler calculations because of the non-anticipating property (see 
Section 2). 3) Stratonovich's interpretation has sounder mathematical and physical bases: a) the ordinary rules of calculus are preserved; b) it is invariant with respect to a time reversal and guarantees the condition of detailed balance [29]; c) in the case of a dynamical system it respects the law of the energy conservation [32]. 4) The kinetic interpretation is the only that agrees with the law of the thermodynamic [11] [27]. 5) The experiments are in accord with Stratonovich's interpretation [20] [21]. 6) The FPK equation deriving from Stratonovich's interpretation has one more term in the drift, the so-called spurious drift that does not originate from a physical reason (it is recalled that this term coincides with the Wong-Zakai-Stratonovich corrective term [33] [34], which makes Itô's solution of the FPK equation equal to Stratonovich's one). In [16] it is supposed that the spurious drift is caused by the infinitely fast fluctuations of the Gaussian white noise. No attempt is made here to give a meaning to the spurious drift.

In writer's thought nothing new in theory can be discovered, but a systematic comparison among the solutions of SDE's according the two points of view lacks in literature. Thus, after introducing the problem in Section 2, in the present research several stochastic dynamic systems with parametric excitations are analyzed comparing Itô's solution with Stratonovich one's. Even if the set of dynamic systems that are analyzed cannot be considered exhaustive, the deep differences in the response statistics are revealed.

\section{Position of the Problem}

Consider the following scalar SDE (generalized Langevin equation)

$$
\dot{X}(t)=a(X(t), t)+g(X(t), t) W(t), \quad X\left(t_{0}\right)=x_{0},
$$

where $W(t)$ is a stationary Gaussian white noise with autocorrelation function $R_{W W}(\tau)=E[W(t) W(t+\tau)]=\delta(\tau)$. The transition probability density function (PDF) $p_{X}$ of the response $X(t)$ is governed by the following FPK equation

$$
\frac{\partial p_{X}}{\partial t}=-\frac{\partial}{\partial x}\left[m_{1}(x) p_{X}\right]+\frac{1}{2} m_{2}(x) \frac{\partial^{2} p_{X}}{\partial x^{2}} .
$$

where $m_{1}(x)$ and $m_{2}(x)$ are the first and the second derivate moment, respectively, which are defined as

$$
\begin{gathered}
m_{1}(x)=\lim _{t \downarrow s}\{E[X(t)-X(s) \mid X(s)=x]\}, \\
m_{2}(x)=\lim _{t \downarrow s}\left\{E\left[(X(t)-X(s))^{2} \mid X(s)=x\right]\right\} .
\end{gathered}
$$

Once the derivate moments are computed, Equation (2) can be solved. The equilibrium solution is

$$
p_{X}(x)=\frac{C}{m_{2}^{2}(x)} \cdot \exp \left[\int \frac{2 m_{1}(x)}{m_{2}^{2}(x)} \mathrm{d} x\right],
$$

where $C$ is a normalization constant. In order Equation (5) to be effectively a PDF, its integral must be finite on the existence domain.

Now, we show that Equation (5) give rise to different solutions according to 
the way in which Equation (1) is interpreted. Recast it in integral form:

$$
X(t)=X\left(t_{0}\right)+\int_{t_{0}}^{t} a(X(t), t) \mathrm{d} t+\int_{t_{0}}^{t} g(X(t), t) \mathrm{d} B(t),
$$

where $B(t)$ is a Brownian motion, and formally or in the sense of the mathematical distributions $\mathrm{d} B / \mathrm{d} t=W(t)$. The serious problem that arises is that the second integral in the right-hand-side is not valuable as a Riemann, Stieltjes or Lebesgue integral because the Brownian motion has unbounded variations in a finite interval of time.

For simplicity's sake let $G(t)=g(X(t), t)$, which is a stochastic process, and consider the integral $Y(t)$ :

$$
Y(t)=\int_{t_{0}}^{t} G(s) \mathrm{d} B(s) .
$$

We divide the time interval $\left[t_{0}, t\right]$ in sub-intervals $\Delta t=t_{i}-t_{i-1}$, and we try to obtain the integral (7) as the limit of the integral sum:

$$
S_{n}=\sum_{i=1}^{n} G\left(t_{k}^{\prime}\right)\left[B\left(t_{i}\right)-B\left(t_{i-1}\right)\right],
$$

where $t_{k}^{\prime} \in\left[t_{i-1}, t_{i}\right]$. Taking the limit of $S_{n}$ for $\Delta_{n} \rightarrow 0$, it should be

$$
Y(t)=\int_{t_{0}}^{t} G(s) \mathrm{d} B(s)=\lim _{\Delta_{n} \rightarrow 0} S_{n} .
$$

Unfortunately, the limit depends on the choice of the point $t_{k}^{\prime}$ [35]. In other words, the integral (7) can take infinite values. Itô [8] selected

$$
t_{k}^{\prime}=t_{i-1} \text {. }
$$

The important consequence of this choice is that any non-anticipating function $G(X(t))$ of the response is uncorrelated with the increment $\mathrm{d} B$ of the Wiener process, that is

$$
E[G \mathrm{~d} B]=E[G] \cdot E[\mathrm{~d} B]=0 .
$$

On the contrary, Stratonovich [34] adopted the midpoint of the interval as $t_{k}^{\prime}$, say

$$
t_{k}^{\prime}=\frac{t_{i-1}+t_{i}}{2} .
$$

Clearly, the non-anticipating property does not hold any longer.

Returning to the derivate moments $(3,4)$, the interpretation of the stochastic integral (7) causes an important difference. We renounce to report their derivation, which is long and tedious, referring to [12] [13].

The final expressions are:

$$
\begin{gathered}
m_{1}^{(I)}=a(x) . \\
m_{1}^{(S)=} a(x)+\frac{1}{2} g(x) \frac{\partial g(x)}{\partial x} . \\
m_{2}=g^{2}(x),
\end{gathered}
$$

where (I) and (S) in the above equations stand for Itô and Stratonovich, respec- 
tively. Comparing Equations (13) and (14), it can be concluded that: 1) the equilibrium solutions (5) are different according to the two interpretations; 2) if the SDE in Equation (1) is modified as

$$
\dot{X}(t)=a(X(t), t)+\frac{1}{2} g(x) \frac{\partial g}{\partial x}+g(X(t), t) W(t),
$$

its Itô's solution equates the Stratonovich's solution. This result was obtained separately by Wong and Zakai [33] and by Stratonovich [34].

By putting $t_{k}^{\prime}=(1-\alpha) t_{i-1}+\alpha t_{i}(0 \leq \alpha \leq 1)$ in Equation (8), infinite prescriptions for the stochastic integral are obtained, and so infinite FPK equations [29] [30] [31]. For brevity's sake, only the cases $\alpha=0$ (Itô) and $\alpha=1 / 2$ will be considered here.

\section{Examples}

In this section the equilibrium PDF's of some scalar SDE's and of one two-state SDE will be computed according Itô's and Stratonovich's prescrition. The differences among the two solutions will be enlightened.

\subsection{System with Cubic Nonlinearity and Nonlinear Parametric Excitation}

We analyze the following nonlinear system

$$
\mathrm{d} X(t)=-\left[a X(t)+b X(t)^{3}\right] \mathrm{d} t+\sqrt{c+\sigma^{2} X(t)} \mathrm{d} B,
$$

where $a, b$ and $c$ are real positive constants, and $B(t)$ is a standard Brownian motion. $X(t)$ is allowed to assumed positive values only. According to Equations (13, 14) the first derivate moments is

$$
\begin{gathered}
m_{1}^{(I)}=-a x-b x^{3} . \\
m_{1}^{(S)}=-a x-b x^{3}+\frac{\sigma^{2}}{4} .
\end{gathered}
$$

in Itô's and Stratonovich's prescriptions, respectively. The third term in Equation (19) equates the Wong-Zakai-Stratonovich corrective term. The second derivate moment is $c+\sigma^{2} x$ in both cases.

According to Itô the equilibrium PDF is

$$
\begin{aligned}
p_{X}^{(I)}(x)= & \frac{C_{I}}{c+\sigma^{2} x} \cdot \exp \left[-\frac{2}{3} \frac{b x^{3}}{\sigma^{2}}+\frac{c b x^{2}}{\sigma^{4}}-\frac{2 a x}{\sigma^{2}}-\frac{2 b c^{2} x}{\sigma^{6}}\right. \\
& \left.+2\left(\frac{a c}{\sigma^{4}}+\frac{b c^{3}}{\sigma^{8}}\right) \ln \left(c+\sigma^{2} x\right)\right],
\end{aligned}
$$

where $C_{I}$ is a normalization constant. According to Stratonovich it is obtained

$$
\begin{aligned}
p_{X}^{(S)}(x)= & \frac{C_{S}}{c+\sigma^{2} x} \cdot \exp \left[-\frac{2}{3} \frac{b x^{3}}{\sigma^{2}}+\frac{c b x^{2}}{\sigma^{4}}-\frac{2 a x}{\sigma^{2}}-\frac{2 b c^{2} x}{\sigma^{6}}\right. \\
& \left.+2\left(\frac{1}{4}+\frac{a c}{\sigma^{4}}+\frac{b c^{3}}{\sigma^{8}}\right) \ln \left(c+\sigma^{2} x\right)\right] .
\end{aligned}
$$


The two PDF's are plotted in Figure 1, where the continuous line is the solution according to Itô and the dashed line the solution according to Stratonovich. The parameters take the values $a=1, b=0.5, c=1, \sigma=1$. In this case, the differences are not remarkable though there are. However, the variances are more different: 0.439473 according to Itô, 0.519702 according to Stratonovich. The larger variance of Stratonovich's solution is caused by the extra-term in Equation (19), which has opposite sign to the drift term.

\subsection{Cubic System with Double Well Potential and Nonlinear Parametric Excitation}

We analyze the following nonlinear system

$$
\mathrm{d} X(t)=\left[a X(t)-b X(t)^{3}\right] \mathrm{d} t+\sqrt{c+\sigma^{2} X(t)} \mathrm{d} B,
$$

where $a, b$ and $c$ are real positive constants, and $B(t)$ is a standard Brownian motion. $X(t)$ is allowed to assumed positive values only. This system has been analyzed in many and many papers, but in most cases the excitation was additive. The restoring force derives from the potential function $a x^{2} / 2-b x^{4} / 4$, which has unstable maximum for $x=0$ and two stable minima in $\pm \sqrt{2 a / b}$.

According to Equations $(13,14)$ the first derivate moments is

$$
\begin{gathered}
m_{1}^{(I)}=a x-b x^{3} . \\
m_{1}^{(S)}=a x-b x^{3}+\frac{\sigma^{2}}{4} .
\end{gathered}
$$

in Itô's and Stratonovich's prescriptions, respectively. As in the previous case, the third term in Equation (24) equates the Wong-Zakai-Stratonovich corrective term, and the second derivate moment is $c+\sigma^{2} x$.

If the excitation is external, this system is known to have a bimodal PDF. With the parametric excitation of Equation (22) the PDF's are

$$
\begin{aligned}
p_{X}^{(I)}(x)= & \frac{C_{I}}{c+\sigma^{2} x} \cdot \exp \left[-\frac{2}{3} \frac{b x^{3}}{\sigma^{2}}+\frac{c b x^{2}}{\sigma^{4}}+\frac{2 a x}{\sigma^{2}}-\frac{2 b c^{2} x}{\sigma^{6}}\right. \\
& \left.+2\left(-\frac{a c}{\sigma^{4}}+\frac{b c^{3}}{\sigma^{8}}\right) \ln \left(c+\sigma^{2} x\right)\right], \\
p_{X}^{(S)}(x)= & \frac{C_{S}}{c+\sigma^{2} x} \cdot \exp \left[-\frac{2}{3} \frac{b x^{3}}{\sigma^{2}}+\frac{c b x^{2}}{\sigma^{4}}+\frac{2 a x}{\sigma^{2}}-\frac{2 b c^{2} x}{\sigma^{6}}\right. \\
& \left.+2\left(\frac{1}{4}-\frac{a c}{\sigma^{4}}+\frac{b c^{3}}{\sigma^{8}}\right) \ln \left(c+\sigma^{2} x\right)\right] .
\end{aligned}
$$

according to Itô and Stratonovich, respectively. With respect to Equations (20, 21) the terms with a change their signs. The two PDF's are plotted in Figure 2 (Itô continuous line, Stratonovich dashed line): the parameters take the values $a=1, b=0.5, c=1, \sigma=1$. The two density functions look very different: the bimodal characteristic is almost lost in Itô's solution, while is marked in Stratonovich's one. The variances are 1.56060 and 1.81564 in the two cases, respectively with a notable difference. 


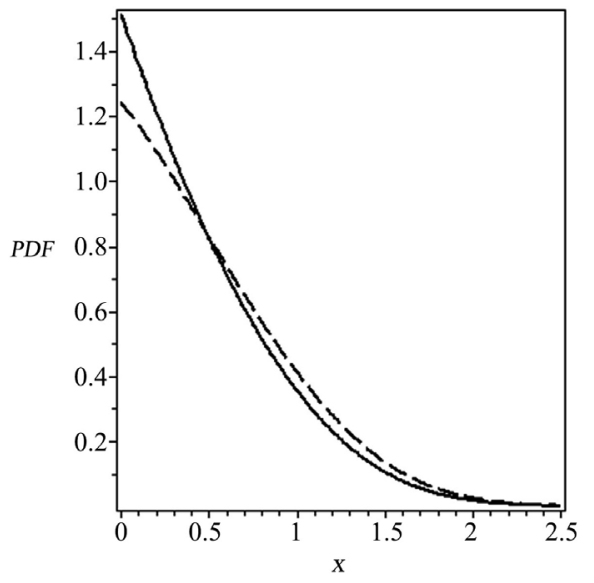

Figure 1. Equilibrium PDF's of the system (17): Itô's solution continuous line (-), Stratonovich solution dashed line (- - - ).

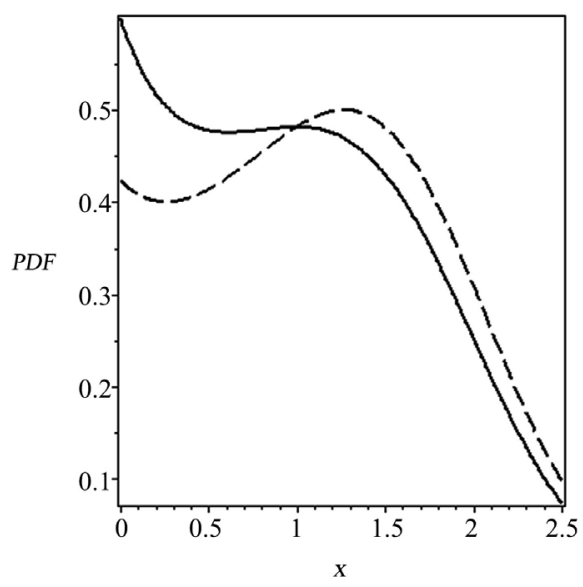

Figure 2. Equilibrium PDF's of the system (22): Itô's solution continuous line (-), Stratonovich solution dashed line (- - - -).

\subsection{Cubic System with Double Well Potential and Strong Nonlinear Parametric Excitation}

The dynamical system of Section 3.2 now has a parametric excitation with a stronger nonlinearity:

$$
\mathrm{d} X(t)=\left[a X(t)-b X(t)^{3}\right] \mathrm{d} t+\sqrt{c+\sigma^{2} X^{2}(t)} \mathrm{d} B,
$$

where $a, b$ and $c$ are real positive constants, and $B(t)$ is a standard Brownian motion. $X(t)$ is allowed to vary on the whole real axis. The first derivate moments is

$$
\begin{gathered}
m_{1}^{(I)}=a x-b x^{3} . \\
m_{1}^{(S)}=a x-b x^{3}+\frac{\sigma^{2}}{2} x .
\end{gathered}
$$

in Itô's and Stratonovich's prescriptions, respectively. As in the previous case, the third term in Equation (29) equates the Wong-Zakai-Stratonovich corrective term, and the second derivate moment is $c+\sigma^{2} x^{2}$.

In this case the PDF's are 


$$
\begin{gathered}
p_{X}^{(I)}(x)=\frac{C_{I}}{c+\sigma^{2} x^{2}} \cdot \exp \left[-\frac{b x^{2}}{\sigma^{2}}+\left(\frac{a}{\sigma^{2}}+\frac{b c}{\sigma^{4}}\right) \ln \left(c+\sigma^{2} x^{2}\right)\right], \\
p_{X}^{(S)}(x)=\frac{C_{S}}{c+\sigma^{2} x^{2}} \cdot \exp \left[-\frac{b x^{2}}{\sigma^{2}}+\left(\frac{1}{2}+\frac{a}{\sigma^{2}}+\frac{b c}{\sigma^{4}}\right) \ln \left(c+\sigma^{2} x^{2}\right)\right] .
\end{gathered}
$$

according to Itô and Stratonovich, respectively.

The PDF's are plotted in Figure 3 for the following values of the parameters: $a=1, b=0.25, c=1$; the strength of the white noise $\sigma$ varies. When the excitation is external, this dynamical system has a bimodal PDF. The behaviour is different with the parametric excitation of Equation (27), as one can see in the plots: the look of the PDF depends on the value of $\sigma$. For values of $\sigma$ lesser than one the PDF is bimodal in both approaches (Figure 3(a)). When $\sigma=1$ (Figure 3(b)), the PDF is unimodal according to Itô, while it is bimodal according to Stratonovich. Hence, according to Stratonovich the system has undergone a phase transition. Raising $\sigma$ to $\sqrt{3}$, both PDF's are bimodal: thus, the phase transition in Itô's interpretation is shifted to values of $\sigma$ a bit larger. Because of the not simple expressions of the PDF's, it is probably impossible to express the zeros of the first derivatives as functions of $\sigma$, but they can be found numerically.

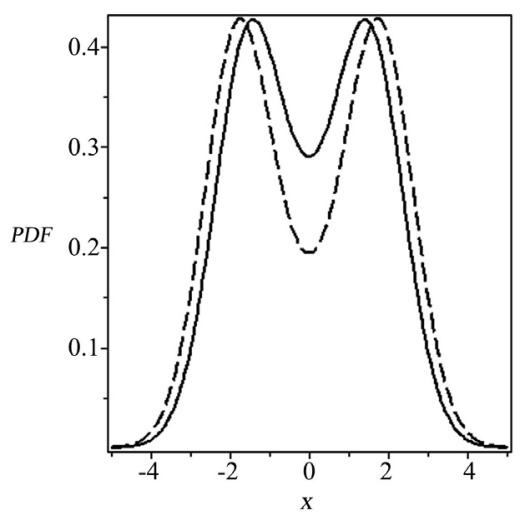

(a)

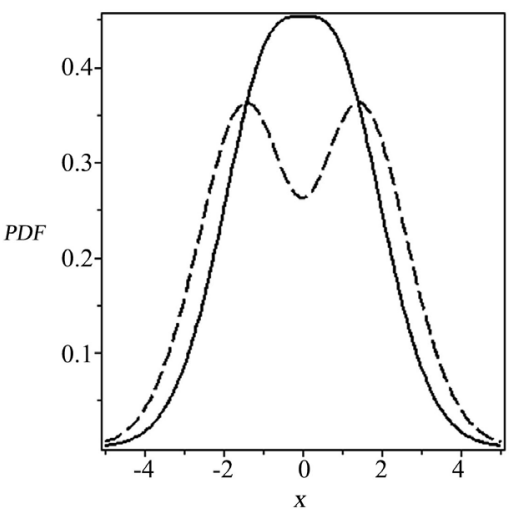

(b)

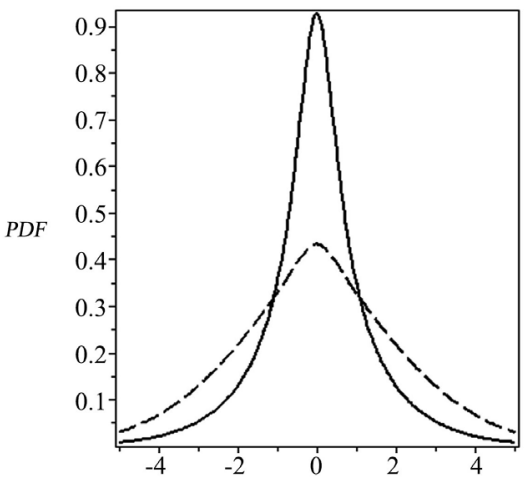

(c)

Figure 3. Equilibrium PDF's of the system (27) for $\sigma=1 / \sqrt{2}$ (a), $\sigma=1$ (b), and $\sigma=\sqrt{3}$ (c): Itô's solution continuous line (-), Stratonovich solution dashed line $(---)$. 
The differences in the PDF's are notable especially in the last case in which they have a maximum only. However, the differences are really deeper as one can see in Table 1, where the variances are reported. In the Stratonovich's approach the variances are much larger than in Itô's one. Moreover, they grow with $\sigma$, while according to Itô they diminish. It is concluded that in this case the two interpretations of the SDE's lead to opposite behaviours of the system.

\subsection{A Two-State System: Nonlinear Oscillator with Parametric Excitation}

Now we consider a nonlinear two-state oscillator with parametric excitations that has been already considered in literature according to the Itô's interpretation of the stochastic integral only [12] [13]:

$$
\ddot{X}(t)+f(X, \dot{X}) \dot{X}(t)+\omega_{0}^{2} X(t)=X(t) W_{1}(t)+\dot{X}(x) W_{2}(t)+W_{3}(t),
$$

where $f(X, \dot{X})$ is a deterministic function of the two states of the oscillator. $W_{1}, W_{2}$ and $W_{3}$ are stationary independent Gaussian white noises with intensities $K_{j j}(j=1,2,3)$, and auto-correlations $R_{W_{j} W_{j}}(\tau)=K_{j j} \delta(\tau)$. The FPK equations associated with Equation (32) are

$$
\begin{gathered}
\frac{\partial p_{X_{1} X_{2}}}{\partial t}=\omega_{0}^{2} \frac{\partial}{\partial x_{1}}\left(x_{1} p_{X_{1} X_{2}}\right)-\frac{\partial}{\partial x_{2}}\left\{\left[-f\left(x_{1}, x_{2}\right) x_{2}\right] p_{X_{1} X_{2}}\right\} \\
+\pi \frac{\partial^{2}}{\partial x_{2}^{2}}\left[\left(K_{11} x_{1}^{2}+K_{22} x_{2}^{2}+K_{33}\right)\right], \\
\frac{\partial p_{X_{1} X_{2}}}{\partial t}=\omega_{0}^{2} \frac{\partial}{\partial x_{1}}\left(x_{1} p_{X_{1} X_{2}}\right)-\frac{\partial}{\partial x_{2}}\left\{\left[-f\left(x_{1}, x_{2}\right) x_{2}+\pi K_{22} x_{2}\right] p_{X_{1} X_{2}}\right\} \\
+\pi \frac{\partial^{2}}{\partial x_{2}^{2}}\left[\left(K_{11} x_{1}^{2}+K_{22} x_{2}^{2}+K_{33}\right)\right],
\end{gathered}
$$

according to Itô and Stratonovich, respectively. Equation (34) differs from Equation (33) because of the term $\pi K_{22} x_{2}$ in the derivative with respect to $x_{2}$.

In both cases the equilibrium PDF, if existent, has the form

$$
p_{X_{1} X_{2}}\left(x_{1}, x_{2}\right)=C \cdot \exp \left[-\phi\left(x_{1}, x_{2}\right)\right] .
$$

In Equation (35) $\phi\left(x_{1}, x_{2}\right)$ is the probability potential that is solution to the first order ODE

$$
\frac{\mathrm{d} \phi}{\mathrm{d} \lambda}=\frac{f\left(x_{1}, x_{2}\right)+\left(\pi K_{22}\right)}{\pi\left(K_{11} x_{1}^{2}+K_{22} x_{2}^{2}+K_{33}\right)} .
$$

Table 1. Variances for the system (27).

\begin{tabular}{ccc}
\hline$\sigma$ & Itô & Stratonovich \\
\hline $1 / \sqrt{2}$ & 5.6363 & 7.2070 \\
1 & 5.0122 & 7.6866 \\
$\sqrt{3}$ & 3.9604 & 9.6512 \\
\hline
\end{tabular}


In Equation (36) the term in parenthesis in the numerator is absent in Itô's interpretation; $\lambda$ is a value of the mechanical energy $\Lambda$ of the oscillator, that is $\Lambda=\frac{1}{2} \omega_{0}^{2} X_{1}^{2}+\frac{1}{2} X_{2}^{2}$. An admissible solution can be found if and only if the following conditions hold [12] [13]

$$
f\left(x_{1}, x_{2}\right)=f(\lambda), \quad K_{11}=\omega_{0}^{2} K_{22} .
$$

The second condition in Equation (37) imposes a rather restrictive relationship among the intensities of two excitations and a system parameter. Assuming that the conditions of Equation (37) are satisfied, the resulting PDF's are

$$
\begin{gathered}
p_{X_{1} X_{2}}^{(I)}\left(x_{1}, x_{2}\right)=C_{I} \cdot \exp \left[-\int \frac{f(\lambda)}{\pi\left(2 K_{22} \lambda+K_{33}\right)} \mathrm{d} \lambda\right], \\
p_{X_{1} X_{2}}^{(S)}\left(x_{1}, x_{2}\right)=\frac{C_{S}}{\sqrt{2 K_{22} \lambda+K_{33}}} \cdot \exp \left[-\int \frac{f(\lambda)}{\pi\left(2 K_{22} \lambda+K_{33}\right)} \mathrm{d} \lambda\right],
\end{gathered}
$$

according to Itô and Stratonovich, respectively.

For brevity's sake, only the case $f(\lambda)=a \lambda$ is presented. The integral in the exponentials is evaluated as

$$
\int \frac{a \lambda}{\pi\left(2 K_{22} \lambda+K_{33}\right)} \mathrm{d} \lambda=\frac{a \lambda}{2 \pi K_{22}}-\frac{a K_{33} \ln \left(2 K_{22} \cdot \lambda+K_{33}\right)}{4 \pi K_{22}} .
$$

In the numerical analyses the parameters take the values: $a=0.1, \omega_{0}=2 \pi, K_{22}=0.1$ in the first case, and $K_{22}=1.0$ in the second $\left(K_{11}\right.$ comes from the second of Equations (37)). The results of the first case are plotted in Figure 4 and Figure 5. Figure 4 shows the two-dimensional PDF given by Equation (38), say Itô's interpretation and the two-dimensional PDF given by Equation (39) (Stratonovich's). One can note that Itô's PDF is rather flat around the maximum, while Stratonovich's one is much more peaked. This fact is confirmed by considering the sections $X_{2}=0, p_{X_{1} \mid X_{2}=0}^{(I)}$ or $p_{X_{1} \mid X_{2}=0}^{(S)}$ and $X_{1}=0, p_{X_{2} \mid X_{1}=0}^{(I)}$ or $p_{X_{2} \mid X_{1}=0}^{(S)}$ that are shown in Figure 5. It is recalled that, strictly speaking, these functions are not PDF's as they are not normalized: however, the normalization could be easily obtained numerically. A measure of the dispersion is the mean square value, that is $\int x_{1}^{2} p_{X_{1} \mid X_{2}=0}^{(\cdot)} \mathrm{d} x_{1}$ or $\int x_{2}^{2} p_{X_{2} \mid X_{1}=0}^{(\cdot)} \mathrm{d} x_{2}$. The dispersions of the section $X_{1}=0$ are 0.030817 and 0.027244 according to Itô and Stratonovich, respectively. The dispersions of the section $X_{2}=0$ are 7.644173 and 6.757889 according to Itô and Stratonovich, respectively. These values are not so different, and partly disavow the visible impression. However, on the whole the two PDF's are different even if less than in other cases.

The results of the second case are in Figure 6, Figure 7: in Figure 6 there are the 3D plots of the joint PDF's, while the sections $X_{2}=0, X_{1}=0$ are in Figure 7. As expected, the differences between the two interpretations of the stochastic differential Equation (32) are more marked in this case. In fact, the strengths $K_{11}, K_{22}$ of the parametric excitations are definitely larger: in this way, the term $\pi K_{22} x_{2}$ in Equation (34) becomes more and more important (this term is ab- 
sent in Itô's interpretation). Even the mean square values are notably different: for the section $X_{2}=0$ they are 0.080475 and 0.055596 according to Itô and to Stratonovich, respectively. For the section $X_{1}=0$ the mean square values are 19.9617 and 13.7905, respectively. Performing the normalization, the variances are $\sigma_{X}^{2}=1.6038$ and 0.8817 according to Itô and Stratonovich, respectively with more notable differences.

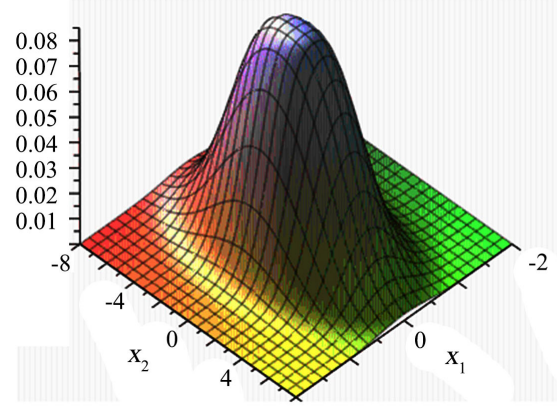

(a)

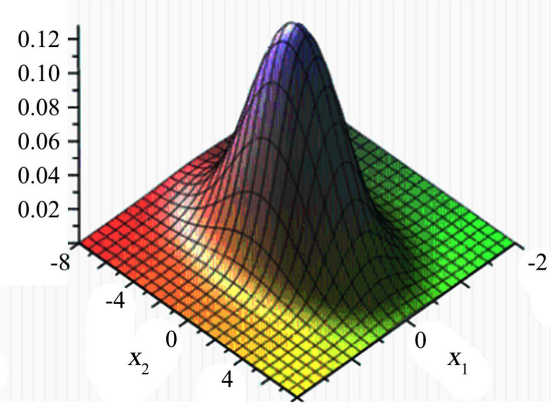

(b)

Figure 4. Two-dimensional PDF of the system (32) according to Itô, first case, Equation (38) (a), and according Stratonovich, Equation (39) (b).

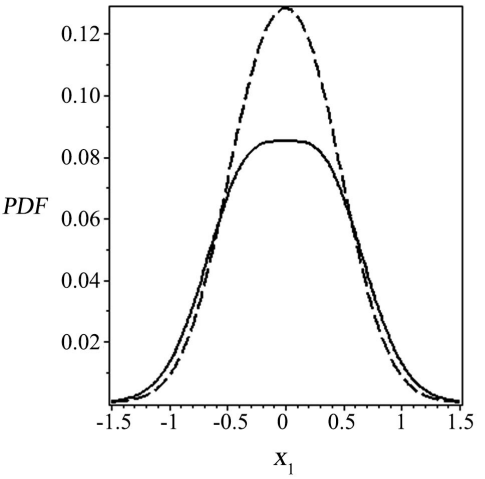

(a)

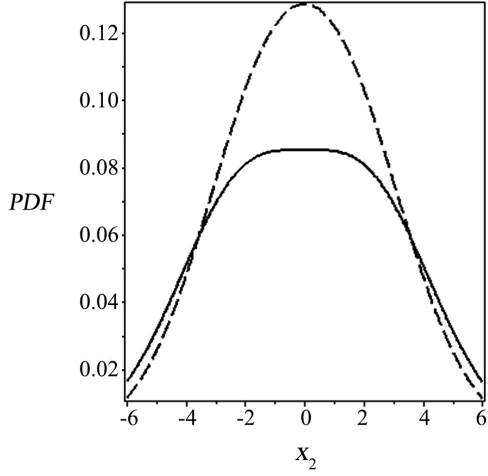

(b)

Figure 5. Sections $X_{2}=0$ (a) and $X_{1}=0$ (b) of the two-dimensional PDF's of the first case: Itô's solution continuous line (-), Stratonovich solution dashed line (- - - -).

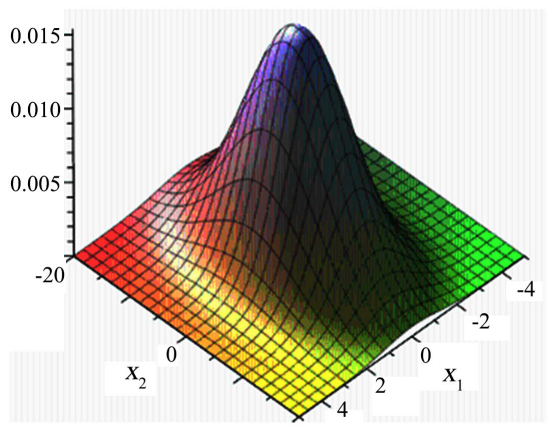

(a)

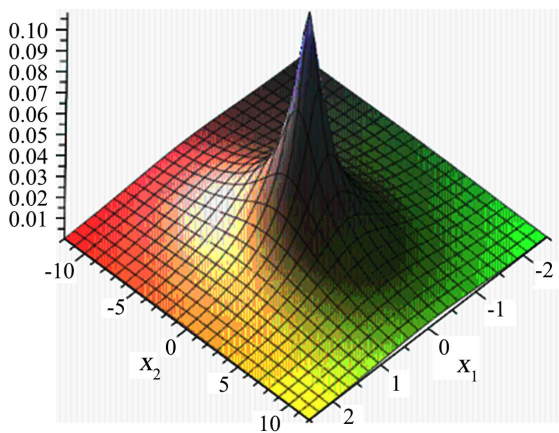

(b)

Figure 6. Two-dimensional PDF of the system (32) second case $K_{22}=1.0$, according to Itô, (Equation (38) (a) and according to Stratonovich (b). 

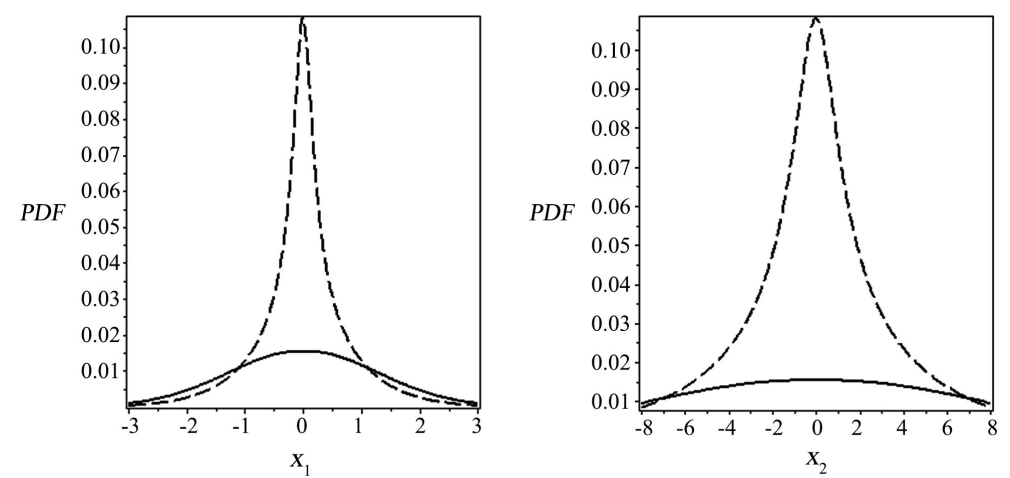

Figure 7. Sections $X_{2}=0$ (a) and $X_{1}=0$

(b) of the two-dimensional PDF's of the second case, $K_{22}=1.0$ : Itô's solution continuous line (-), Stratonovich solution dashed line (- - ) .

Another important aspect of the behaviour of a dynamic system is the mean upcrossing rate function $v_{X}(b)$, that is the function that counts the times a stochastic process overpasses a given threshold $b$ with positive velocity. For a second order system this function is given by Rice's formula [35]:

$$
v_{X}(b)=\int_{0}^{+\infty} p_{X_{1} X_{2}}\left(x_{1}=b, x_{2}\right) \mathrm{d} x_{2} .
$$

If one is interested in the outcrossings of the double barrier $\pm b$ and the PDF $p_{X_{1} X_{2}}$ is symmetric, it is sufficient to double the result of Equation (41). In general, this is to be evaluated numerically.

In the case of high barriers, the upcrossings are rare events so that they can be considered independent and are assumed to constitute a homogeneous Poisson process. Thus, the cumulative distribution function of the first time $T(b)$ at which the process $X(t)$ crosses $b$ firstly is given by

$$
P[T(b) \leq \tau]=F_{T}(\tau)=1-\mathrm{e}^{-v_{X}(b) \tau} .
$$

From the usual laws of the probability it is obtained the important result

$$
P[\max X(t) \mid(0, \tau] \leq b]=\mathrm{e}^{-v_{X}(b) \tau} .
$$

Thus, the knowledge of the mean upcrossing rate function yields an estimate of the largest value distribution of $X(t)$ : the estimate is as more precise as the upcrossings can be considered Poissonian.

As an example, Equation (41) has been numerically evaluated for the oscillator of Equation (32) in the second case, that is with $K_{22}=1.0$, considering several values of $b$ : the results are in Figure 8, where the dashed line comes from Itô's interpretation and the continuous line from Stratonovich's one. The two curves are markedly different: in Itô's interpretation for a given barrier $b$ there are roughly twice upcrossings than in Stratonovich's one, what means that the probability of reaching and passing a given response level is higher according to Itô. This fact is surely relevant in structural design. The explanation of this behaviour resides in the extra-term in the drift that arises in Stratonovich interpretation: in the case under consideration it makes the dynamic system stiffer so that crossing a given level becomes rarer. 


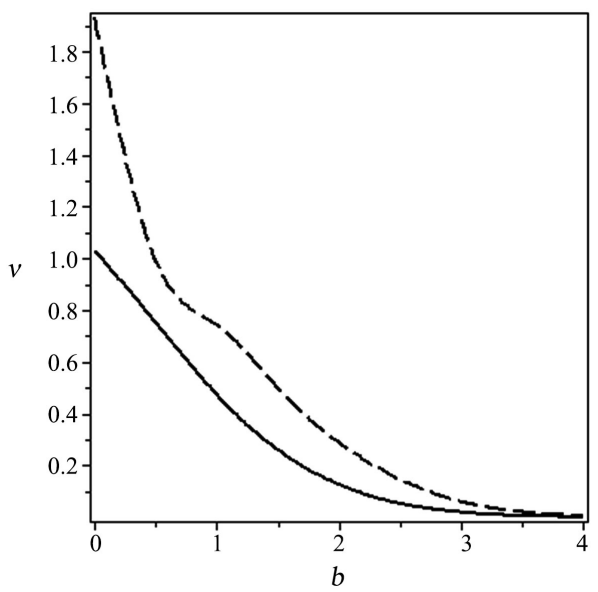

Figure 8. Plot of the mean upcrossing rate function $v(b)$ against the barrier $b$ for the oscillator of Equation (32) with $K_{22}=1.0$ : Itô's solution continuous line (-), Stratonovich solution dashed line (- - - -).

\section{Conclusions}

In this paper, the problem of the interpretation of the stochastic differential equations is addressed from an applicative point of view. Theoretically, when evaluating a stochastic integral with respect to a Brownian motion, there are infinite interpretations, and hence infinite values for the integral depending on the choice of the integration point. However, Itô's interpretation and Stratonovich's one are the most common so that attention is restricted to these two interpretations, even if the kinetic interpretation too is relevant. It has been underlined [36] that the interpretation of a stochastic differential equation has a deep deterministic root, residing in the integration rule that one adopts: Itô's choice corresponds to the forward integration rule, while Stratonovich suggests the trapezoidal rule.

In order to highlight the differences in the responses that are obtained according the two interpretations of a stochastic integral, some stochastic dynamic systems with parametric excitation are analyzed, for all of which the FPK equation has an analytical solution in the final equilibrium regime. In the first three cases, the system is scalar. In the case of Section 3.1, the restoring force derives from a potential function with a minimum only: there are small differences between the PDF's of the two approaches, which however are present.

In the cases that are examined in Sections 3.2 and 3.3, the potential function has two stable minima and an unstable maximum in zero. When the excitation is merely external, the equilibrium PDF is always bimodal. It remains bimodal in both interpretations in the case of Section 3.2 that is characterized by a milder parametric excitation $\left(\sqrt{c+\sigma^{2} X^{2}} \mathrm{~d} B\right)$, but the PDF's look very differently.

In Section 3.3, the excitation is definitely more nonlinear $\left(\sqrt{c+\sigma^{2} X^{2}} \mathrm{~d} B\right)$, and the behaviour changes dramatically as the PDF may be unimodal or bimodal: see Figure 3. In the two approaches, the phase transition happens for different values of the parameter $\sigma$. The differences between the PDF's increase as $\sigma$ increases, which is reflected by very different values of the mean square $\left\langle X^{2}\right\rangle$ 


\section{(Table 1).}

The last case (Section 3.4) regards a second order oscillator, for which the problem of the mean upcrossing rate of a given level is also addressed. The responses are not comparable, and the oscillator is safer according to Stratonovich than according to Itô.

The following conclusions can be drawn: 1) the two interpretations of a stochastic integral lead to marked differences in the statistical features of the response of a dynamic system. 2) Hence, caution is necessary in choosing an interpretation instead of the other. 3) Likely, Stratonovich's interpretation is preferable as it has deeper physical bases, the respect of the law of the conservation of the energy and the agreement with the experiments. 4) However, one can equally use Itô's stochastic differential calculus by modifying the original SDE with the addition of the Wong-Zakai-Stratonovich corrective term in the drift: in such a way Stratonovich's solution is recovered.

In writer's thought, the future work should follow two lines: 1) execution of experiments on dynamic systems with parametric excitation miming real cases; 2) theoretical studies regarding the effects of the different interpretations of a stochastic integrals on other aspects of the stochastic dynamics such as stochastic stability, first passage time problem, and white but not Gaussian excitations.

\section{Conflicts of Interest}

The author declares no conflicts of interest regarding the publication of this paper.

\section{References}

[1] Einstein, A. (1905) Über die von der molekularkinetischen Theorie der Wärme geforderte Bewegung von in ruhenden Flüssigkeiten suspendierten Teilchen. Annalen der Physik, 322, 549-560. https://doi.org/10.1002/andp.19053220806

[2] Langevin, P. (1908) Sur la théorie du mouvement Brownian. Comptes Rendus de I Académie des Sciences, 146, 530.

[3] Fokker, A. (1914) Die Mittlere Energie Rotierender Elektrischen Dipole in Strahlungsfeld. Annalen der Physik, 348, 810-820. https://doi.org/10.1002/andp.19143480507

[4] Planck, M. (1917) Sitzungsber Preuss Akad. Wiss.Phys. Math. K1, 325.

[5] Kolmogorov, A.N. (1931) Über Die Analytischen Methoden in der Wahrscheinlichkeitsrechnung. Mathematische Annalen, 104, 415-458. https://doi.org/10.1007/BF01457949

[6] Chandrasekhar, S. (1943) Stochastic Problems in Physics and Astronomy. Reviews of Modern Physics, 15, 1. https://doi.org/10.1103/RevModPhys.15.1

[7] Itô, K. (1951) On Stochastic Differential Equations. Nagoya Mathematical Journal, 3, 55-65. https://doi.org/10.1017/S0027763000012216

[8] Stratonovich, R.L. (1963) Topics in the Theory of Random Noise. Gordon Breach, New York.

[9] Hänggi, P. (1978) Stochastic Processes I. Asymptotic Behaviour and Symmetries. Helvetica Physica Acta, 51, 183. 
[10] Klimontovich, Y.L. (1990) Itô, Stratonovich and Kinetic Forms of Stochastic Equations. Physica A, 163, 515-532. https://doi.org/10.1016/0378-4371(90)90142-F

[11] Lin, Y.K. and Cai, G.-Q. (1995) Probabilistic Structural Dynamics: Advanced Theory and Applications. McGraw-Hill, New York.

[12] Cai, G.-Q. and Zhu, W.-Q. (2017) Elements of Stochastic Dynamics. World Scientific, Singapore.

[13] Gray, A.H. and Caughey, T.K. (1965) A Controversy in Problems Involving Random Parametric Excitation. Journal of Mathematics and Physics, 44, 288-296. https://doi.org/10.1002/sapm1965441288

[14] Mortensen, R.E. (1969) Mathematical Problems of Modelling Stochastic Nonlinear Dynamic Systems. Journal of Statistical Physics, 1, 271-296. https://doi.org/10.1007/BF01007481

[15] Ryter, D. (1978) Langevin Equations and Stochastic Integrals. Zeitschrift für Physik B Condensed Matter, 30, 219-222. https://doi.org/10.1007/BF01320988

[16] Srinivasan, S.K. (1978) Stochastic Integrals. SM Archives, 3, 325.

[17] West, B.J., Bulsara, A.R., Lindenberg, K., Seshadri, V. and Shuler, K.E. (1969) Stochastic Processes with Non-Additive Fluctuations: I. Itô and Stratonovich Calculus and the Effects of Correlations. Physica A, 97, 211-233. https://doi.org/10.1016/0378-4371(79)90103-1

[18] van Kampen, N.G. (1981) Itô versus Stratonovich. Journal of Statistical Physics, 24, 175-187. https://doi.org/10.1007/BF01007642

[19] Smyth, J., Moss, F., McClintock, P.V.E. and Clarkson, D. (1983) Itô versus Stratonovich Revisited. Physics Letters A, 97, 95-98. https://doi.org/10.1016/0375-9601(83)90520-0

[20] McClintock, P.V.E. and Moss, F. (1985) Further Experimental Evidence Pertaining to the Applicability of Itô and Stratonovich Stochastic Calculi to Real Physical Systems. Physics Letters A, 107, 367-370. https://doi.org/10.1016/0375-9601(85)90691-7

[21] To, C.W.S. (1988) On Dynamic Systems Disturbed by Random Parametric Excitation. Journal of Sound and Vibration, 123, 387-390. https://doi.org/10.1016/S0022-460X(88)80121-4

[22] Di Paola, M. and Falsone, G. (1993) Itô and Stratonovich Integrals for Delta-Correlated Processes. Probabilistic Engineering Mechanics, 8, 197-208. https://doi.org/10.1016/0266-8920(93)90015-N

[23] Caddemi, S. and Di Paola, M. (1996) Ideal and Physical White Noise in Stochastic Analysis. International Journal of Non-Linear Mechanics, 31, 581. https://doi.org/10.1016/0020-7462(96)00023-6

[24] Di Paola, M. and Pirrotta, A. (2004) Direct Derivation of Corrective Terms in SDE through Nonlinear Transformation on Fokker-Planck Equation. Nonlinear $D y$ namics, 36, 349-360. https://doi.org/10.1023/B:NODY.0000045511.89550.57

[25] Braumann, C.A. (2007) Itô versus Stratonovich Calculus in Random Population Growth. Mathematical Biosciences, 206, 81-107. https://doi.org/10.1016/j.mbs.2004.09.002

[26] Mannella, R. and McClintock, P.V.E. (2012) Itô versus Stratonovich: 30 Years Later. Fluctuation and Noise Letters, 11, Article No. 1240010. https://doi.org/10.1142/S021947751240010X

[27] Yuan, R. and Ao, P. (2012) Beyond Itô versus Stratonovich. Journal of Statistical Mechanics. Theory and Experiment, 2012, P07010. 
https://doi.org/10.1088/1742-5468/2012/07/P07010

[28] Gonzales Arenas, Z. and Barci, D.G. (2012) Hidden Symmetries and Equilibrium Properties of Multiplicative White Noise Stochastic Processes. Journal of Statistical Mechanics. Theory and Experiment, 2012, P12005. https://doi.org/10.1088/1742-5468/2012/12/P12005

[29] Hottovy, S., Volpe, G. and Wehr, J. (2012) Noise-Induced Drift in Stochastic Differential Equations with Arbitrary Friction and Diffusion in the Smoluchowski-Kramers Limit. Journal of Statistical Physics, 146, 762-773. https://doi.org/10.1007/s10955-012-0418-9

[30] Aron, C., Barci, D.G., Cugliandolo, L.F., Gonzales Arenas, Z. and Lozano, G.S. (2016) Dynamical Symmetries of Markov Processes with Multiplicative White Noise. Journal of Statistical Mechanics: Theory and Experiment, 2016, Article No. 053207. https://doi.org/10.1088/1742-5468/2016/05/053207

[31] Sun, X., Duan, J. and Li, X. (2016) Stochastic Modelling of Nonlinear Oscillators under Combined Gaussian and Poisson White Noise: A Viewpoint Based on the Energy Conservation Law. Nonlinear Dynamics, 84, 1311. https://doi.org/10.1007/s11071-015-2570-7

[32] Wong, E. and Zakai, M. (1965) On the Convergence of Ordinary Integrals to Stochastic Integrals. The Annals of Mathematical Statistics, 36, 1560-1564.

[33] Stratonovich, R.L. (1966) A New Representation for Stochastic Integrals and Equations. SIAM Journal on Control, 4, 362-371. https://doi.org/10.1137/0304028

[34] Gardiner, C.W. (2004) Handbook of Stochastic Methods: For Physics, Chemistry and Natural Sciences. Springer, Berlin.

[35] Rice, S.O. (1944) Mathematical Analysis of Random Noise. The Bell System Technical Journal, 23, 282-332. https://doi.org/10.1002/j.1538-7305.1944.tb00874.x

[36] Di Paola, M. (1993) Stochastic Differential Calculus. In: Casciati, F. Ed., Dynamic Motion: Chaotic and Stochastic Behaviour, Springer-Verlag, Vienna, 29-92. https://doi.org/10.1007/978-3-7091-2682-0_2 